\title{
SL2-like spliced leader RNAs in the basal nematode Prionchulus punctatus: New insight into the evolution of nematode SL2 RNAs
}

\author{
NEALE HARRISON, ${ }^{1}$ ANDREAS KALBFLEISCH, ${ }^{1,2}$ BERNADETTE CONNOLLY, JONATHAN PETTITT, \\ and BERNDT MÜLLER \\ School of Medical Sciences, Institute of Medical Sciences, University of Aberdeen, Aberdeen AB25 2ZD, Scotland, United Kingdom
}

\begin{abstract}
Spliced-leader (SL) trans-splicing has been found in all molecularly characterized nematode species to date, and it is likely to be a nematode synapomorphy. Most information regarding SL trans-splicing has come from the study of nematodes from a single monophyletic group, the Rhabditida, all of which employ SL RNAs that are identical to, or variants of, the SL1 RNA first characterized in Caenorhabditis elegans. In contrast, the more distantly related Trichinella spiralis, belonging to the subclass Dorylaimia, utilizes a distinct set of SL RNAs that display considerable sequence diversity. To investigate whether this is true of other members of the Dorylaimia, we have characterized SL RNAs from Prionchulus punctatus. Surprisingly, this revealed the presence of a set of SLs that show clear sequence similarity to the SL2 family of spliced leaders, which have previously only been found within the rhabditine group (which includes $C$. elegans). Expression of one of the $P$. punctatus SL RNAs in $C$. elegans reveals that it can compete specifically with the endogenous $C$. elegans SL2 spliced leaders, being spliced to the pre-mRNAs derived from downstream genes in operons, but does not compete with the SL1 spliced leaders. This discovery raises the possibility that SL2-like spliced leaders were present in the last common ancestor of the nematode phylum.
\end{abstract}

Keywords: gene expression; nematode; phylogeny; spliced leader RNA; spliced leader trans-splicing

\section{INTRODUCTION}

Spliced leader trans-splicing involves the modification of the $5^{\prime}$ ends of pre-mRNAs via an intermolecular splicing event, which results in the addition of a short RNA, the spliced leader (SL), to mRNAs (Hastings 2005). The SL is donated by the independently transcribed SL RNA and targets an unpaired splice acceptor site near the $5^{\prime}$ end of the target pre-mRNA. SL RNAs contain two functionally distinct domains: an SL exon and an intron that includes the Sm sequence motif, a binding site for Sm proteins involved in cis-splicing (Mattaj 1988). They also generally

\footnotetext{
${ }^{1}$ These authors contributed equally to this work.

${ }^{2}$ Present address: Institute of Biochemistry, Justus-Liebig-University Giessen, 35392 Giessen, Germany.

Reprint requests to: Jonathan Pettitt, School of Medical Sciences, Institute of Medical Sciences, University of Aberdeen, Aberdeen AB25 2ZD, Scotland, United Kingdom; e-mail. j.pettitt@abdn.ac.uk; fax: 44-1224555844; or Berndt Müller, School of Medical Sciences, Institute of Medical Sciences, University of Aberdeen, Aberdeen AB25 2ZD, Scotland, United Kingdom; e-mail: b.mueller@abdn.ac.uk; fax: 44-1224555844.

Article published online ahead of print. Article and publication date are at http://www.rnajournal.org/cgi/doi/10.1261/rna.2155010.
}

conform to a similar structure consisting of three stemloops, two of which flank the Sm sequence motif. However, there is considerable sequence diversity, both in terms of length and content. There is no obvious conserved function for SL trans-splicing, although in some groups of organisms functions have been clearly assigned, including the regulation of mRNA stability and translation and the resolution of polycistronic RNAs (Davis and Hodgson 1997; Zeiner et al. 2003; Lall et al. 2004; Blumenthal 2005; Satou et al. 2006; Marletaz et al. 2008; Wallace et al. 2010).

The range of organisms in which SL trans-splicing has been identified is broad, extending from protists through to chordates (Sutton and Boothroyd 1986; Tessier et al. 1991; Davis 1997; Stover and Steele 2001; Blumenthal 2005; Pouchkina-Stantcheva and Tunnacliffe 2005; Zayas et al. 2005; Guiliano and Blaxter 2006; Zhang et al. 2007; Marletaz et al. 2008; Pettitt et al. 2008; Zhang and Lin 2009; Derelle et al. 2010; Douris et al. 2010; Matsumoto et al. 2010), although it appears to be absent from plants, fungi, and vertebrates. The phylogenetic distribution of SL transsplicing is sporadic, however, and does not allow one to distinguish whether this distribution reflects a single origin 
of SL trans-splicing followed by multiple independent losses or whether the process has arisen through multiple, independent events in the different lineages in which it occurs. The similarity of SL RNA structure would seem to support a common, divergent origin for SL trans-splicing. It is also consistent with the hypothesis that snRNAs associated with splicing, in particular U1 snRNA, have the potential to mutate into SL RNAs, explaining both the conserved features of SL RNAs and their sporadic appearance throughout eukaryotic evolution.

From the perspective of the evolution of SL transsplicing, the nematodes are an important group, since it seems likely that this process was present in the last common ancestor of the phylum. Most of what we know about nematode SL trans-splicing is derived from a single species, Caenorhabditis elegans, in which there are two SL RNAs that participate in distinct SL trans-splicing events: SL1 RNA is employed in all cases where a pre-mRNA possesses a $5^{\prime}$ untranslated region ( $5^{\prime} \mathrm{UTR}$ ) that includes an unpaired splice acceptor site. In contrast, SL2 RNA is spliced onto mRNAs whose 5 ' ends are formed during the processing of polycistronic operon transcripts into monocistronic mRNAs for translation (Spieth et al. 1993).
SL1 and closely related SL1-like molecules have been identified in other nematodes. However, all of these fall within the same monophyletic group as C. elegans, termed the Rhabditida (Fig. 1A; Guiliano and Blaxter 2006; Holterman et al. 2006; Meldal et al. 2007). In contrast, the distribution of SL2 RNAs appears to be more limited, being found only in the clade of nematodes that includes C. elegans, the Rhabditina (Guiliano and Blaxter 2006), suggesting that this class of SL RNAs evolved relatively late in nematode evolution.

An important question is the extent to which this view of SL trans-splicing can be applied to the less well-studied members of the phylum. This question has recently been addressed with the availability of the draft genome sequence from Trichinella spiralis. This nematode occupies a significant phylogenetic position, being a member of the Dorylaimia clade, which branches close to the base of the nematode phylum (Fig. 1A; Holterman et al. 2006; Meldal et al. 2007). Members of this basal clade display molecular, cellular, and embryological traits found in other animal groups that appear to have been lost in the Rhabditida (Lavrov and Brown 2001; Bürglin 2008; Heger et al. 2009; Schulze and Schierenberg 2009).
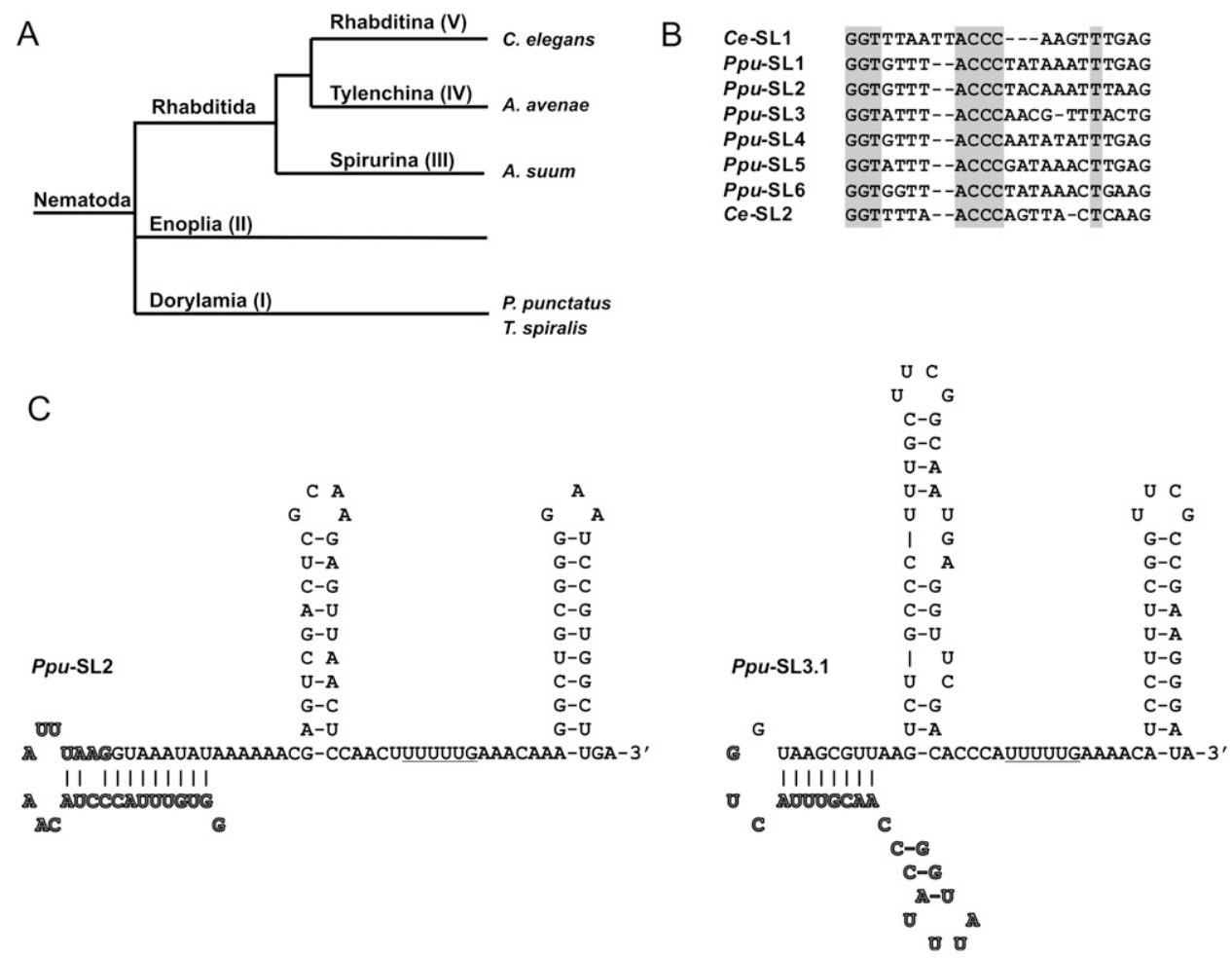

FIGURE 1. $P$. punctatus SL sequences are related to SL1 and SL2. (A) A simplified nematode phylogeny based on ribosomal RNA sequences showing clades I-V (Holterman et al. 2006; Meldal et al. 2007). Species indicated are Caenorhabditis elegans, Aphelenchus avenae, Ascaris suum, Prionchulus punctatus, and Trichinella spiralis. (B) Alignment of P. punctatus Ppu-SL1 to Ppu-SL6 spliced leader sequences with C. elegans SL1 and SL2 spliced leaders. Conserved sequence elements outside the splice junction are boxed in gray. (C) Proposed secondary structures of $P p u$-SL2 and Ppu-SL3.1 RNAs (GenBank entries GU936306 and GU936307) produced using M-fold (Zuker 2003). The SL sequence is shown outlined, and the putative Sm sequence motif is underlined. 
We have previously shown that the canonical SL1 transsplicing found in members of the Rhabditida does not appear to be present in T. spiralis (Pettitt et al. 2008). Rather, the SL RNAs from this nematode showed unprecedented levels of sequence diversity and could not be readily placed in either of the SL1 or SL2 families previously described. We proposed two possible scenarios to explain these data. First, the ancestral nematode resembled T. spiralis, having a diverse array of distinct SL genes, which in the lineage leading the Rhabditida underwent homogenization events leading to the presence of SL1 and SL1-like RNA genes. Alternatively, a single ancestral SL RNA gene that resembled SL1 underwent successive rounds of gene duplication and diversification events in the lineage leading to T. spiralis (Pettitt et al. 2008).

To attempt to distinguish between these possibilities, we have investigated SL trans-splicing in another dorylaimid nematode, Prionchulus punctatus. Surprisingly the SLs that we identified from this nematode do not closely resemble those from T. spiralis. Rather they resemble the SL2 family that has previously only been identified in Rhabditina nematodes (Guiliano and Blaxter 2006). Consistent with this, we show that one of these SL2-like molecules, when expressed in C. elegans, can specifically participate in SL2mediated SL trans-splicing but is not apparently added to genes whose pre-mRNAs are matured via SL1 transsplicing. This discovery raises questions about the likely nature of SL trans-splicing in the last common ancestor of the nematode phylum.

\section{RESULTS}

From a random sample of $P$. punctatus cDNA clones, prepared by $5^{\prime}$ RACE using oligo-dT as a primer, we identified a cDNA sequence corresponding to a gene encoding a vacuolar protein, hereafter designated $P p u-v h a-1$. The $5^{\prime}$ end of the Ppu-vha-1 cDNA was similar in terms of size and nucleotide content to the SL2 family of SLs found in C. elegans and other members of the Rhabditina (Guiliano and Blaxter 2006). We designated this putative SL Ppu-SL1 (Fig. 1B). However, in the absence of genomic sequence, we were unable to directly verify that this sequence was not simply a chance match to the SL sequences. We reasoned that if this sequence arose from the trans-splicing of a $P$. punctatus SL sequence, then we would expect to find it at the $5^{\prime}$ ends of mRNAs derived from additional genes. To identify such genes, we used an oligonucleotide corresponding to the putative SL in $3^{\prime}$ RACE reactions. In this way, we identified cDNAs corresponding to seven different $P$. punctatus genes (Table 1). The presence of this same sequence at the $5^{\prime}$ ends of multiple different mRNAs made it highly likely that we had identified a bona fide SL in P. punctatus.

To attempt to identify additional SLs in $P$. punctatus, we carried out $5^{\prime}$ RACE using a $P p u$-vha-1 gene-specific primer. This allowed us to sample the range of SLs trans-
TABLE 1. Prionchulus punctatus CDNA sequences with Ppu-SL1 at their $5^{\prime}$-ends

\begin{tabular}{llc}
\hline Gene & \multicolumn{1}{c}{ Predicted product } & GenBank entries \\
\hline Ppu-vha-1 & Vacuolar protein & GU936298 \\
Ppu-rpl-36 & 60S Ribosomal protein L36 & GU936300 \\
Ppu-gst-1 & Glutathione-S-transferase & GU936301 \\
Ppu-ubc-2 & Ubiquitin-conjugating enzyme & GU936303 \\
Ppu-ger-1 & GDP-keto-6-deoxymannose & GU936302 \\
& 3,5-epimerase/4-reductase & GU936305 \\
Ppu-rps-15 & Ribosomal protein S15 & GU936304 \\
Ppu-ubl-1 & Ubiquitin/ribosomal protein S27a & \\
\hline
\end{tabular}

These cDNA sequences were obtained by $3^{\prime}$ RACE using the PpuSL1 primer in combination with 3' RACE primers.

ancomplete sequence.

spliced onto the pre-mRNA of this gene, and we were thus able to identify three additional putative SLs (Ppu-SL2, -SL3, and -SL4) that differed from the original sequence in only a few positions (Fig. 1B, Supplemental Table 1). The fact that the sequence variations were only observed in the putative SL sequences, and that most variations were sequenced in several independent $5^{\prime} \mathrm{RACE}$ products, suggested that these were not the result of PCR-induced mutations but represented different SL variants.

To rule out the possibility that the SL-carrying cDNAs were amplified from the $R$. dolichura prey worms that we use to culture $P$. punctatus, we showed that Ppu-vha-1 could be PCR amplified from lysates of single P. punctatus animals but not from the prey worms (Supplemental Fig. 1). Similarly, phylogenetic analyses using the coding sequences derived from the SL-cDNA sequences reported here showed clear affinity between $P$. punctatus and other dorylaimid species to the exclusion of nematodes from the Rhabditida clade (Supplemental Fig. 2).

Since all known SLs are derived from longer SL RNAs, to provide further evidence that these moieties were the products of SL trans-splicing, we attempted to clone the predicted precursor SL RNAs via poly(A) tailing of total $P$. punctatus RNA. We chose two SLs (Ppu-SL2 and Ppu-SL3) to apply this technique to, and we identified cDNAs of $\sim 100$ nucleotides (nt) (Fig. 1C) for each one. While we identified one transcript with $P p u$-SL2, we found two $P p u$ SL3 containing RNAs (Ppu-SL3.1, -SL3.2) with subtle but significant sequence variations (Supplemental Table 1A). These sequences are predicted to form three stem-loops: one contains the SL splice site, and the other two flank an Sm sequence motif, as has previously been observed for all other nematode SL RNAs (Fig. 1C). Sequencing of the products of the $5^{\prime} \mathrm{RACE}$ experiments, using gene-specific primers located in the SL RNA introns for both SL RNA genes, confirmed the SL RNA sequences in each case.

In C. elegans the SL1 RNA genes are clustered in a tandem array (Blumenthal 2005). We reasoned that if this were the case in $P$. punctatus, it might be possible to detect such 
a situation by amplifying from genomic DNA using combinations of primers derived from each SL RNA. Using this approach, we were able to identify two additional SLs (SL5 and SL6) (Fig. 1B) and four additional SL RNA genes (PpuSL1, Ppu-SL5.1, Ppu-SL5.2, and Ppu-SL6) (see Supplemental Table 1). One of the genomic PCR products that we isolated contained three tandem SL RNA genes, showing that at least some of the SL RNA genes are clustered. Unlike the situation in C. elegans, the Ppu-SL RNA genes that we identified did not appear to be interspersed with 5S RNA genes.

Previous studies of rhabditid nematodes have demonstrated the existence of SLs specifically associated with the SL trans-splicing to downstream genes in polycistronic operons (Spieth et al. 1993; Evans et al. 1997; Blumenthal et al. 2002). This SL2 family of SLs can be distinguished from SL1 and SL1-like SLs on the basis of specific sequence motifs present in the SL itself (Guiliano and Blaxter 2006). Comparing the $P p u$-SLs with previously described SLs from other nematodes, it was clear that they could be classified as members of the SL2-like family based upon the spacing between the 5' GGTWW, the central CCCA, and the splice-donor site. In addition, previous studies have identified residues located in the third stem-loop of SL2 RNAs that are essential for SL2 trans-splicing in C. elegans; a GGGG stretch separated from UCCG by 2 or 3 nt (Evans and Blumenthal 2000). Of the Ppu-SL RNAs that we have identified, all but the Ppu-SL3 RNAs have these residues conserved in the loop region of their predicted third stemloops (Fig. 1C; Supplemental Table 1A), providing further support that they are SL2-like SL RNAs.

To provide experimental verification that the $P p u$-SLs fall into the SL2-like family, we investigated whether they are capable of competing with the endogenous C. elegans SLs, and of being trans-spliced onto pre-mRNAs that normally received SLs. We also reasoned that if they are able to participate in SL trans-splicing in C. elegans, then they might show a bias for splicing onto pre-mRNAs derived from downstream genes in operons. To this end, we generated transgenic C. elegans strains expressing Ppu-SL2 and PpuSL3.1 RNAs under the control of a C. elegans U2 RNA gene promoter (Fig. 2A; Ferguson and Rothman 1999).

For both constructs, we tested several independent transgenic lines and assayed for the presence of the PpuSL at the $5^{\prime}$ ends of previously reported trans-spliced $C$. elegans genes. We were unable to detect any messages that received Ppu-SL3.1 in the three transgenic lines produced, and were not able to detect Ppu-SL3.1 RNA using the sensitive method used for the cloning of this RNA from $P$. punctatus RNA preparations. In contrast, trans-splicing of $P p u$-SL2 was observed in the two transgenic lines produced, indicating that $P p u$-SL2 was capable of acting as a substrate for the SL trans-splice reaction in C. elegans.

We first tested a known SL2 trans-spliced gene, Y67H2A.5. Using a gene-specific primer combined with
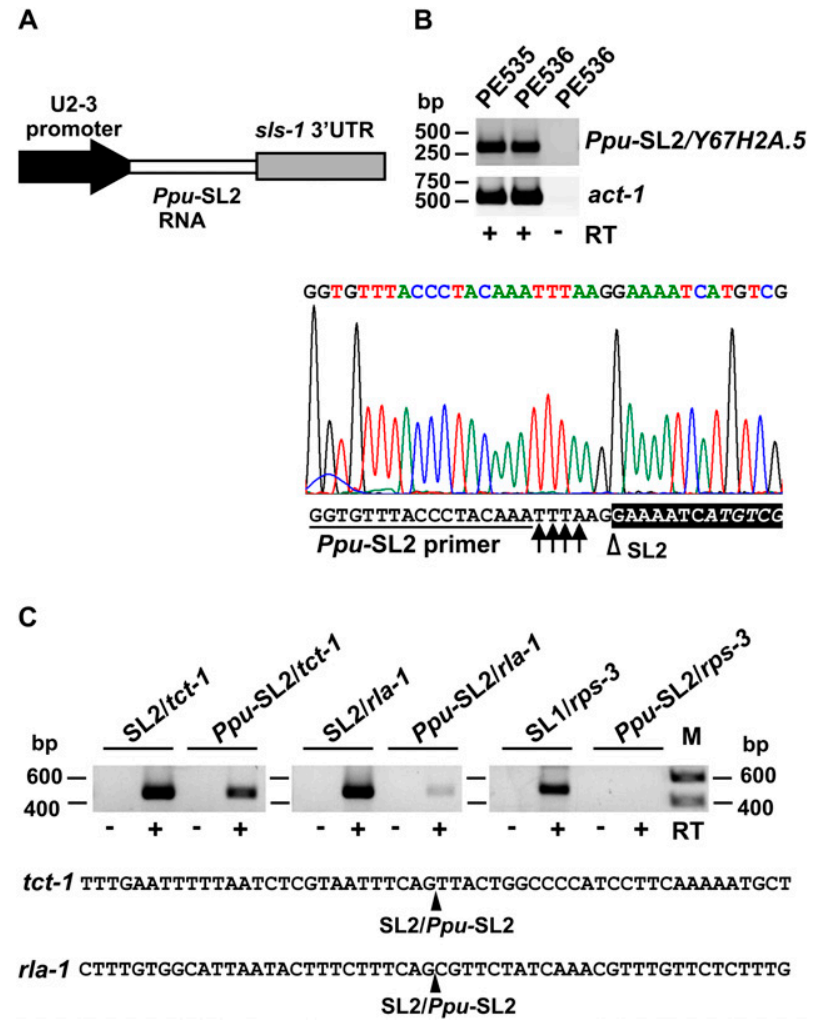

FIGURE 2. $P p u$-SL2 RNA expressed in C. elegans is trans-spliced to SL2 accepting transcripts. (A) Schematic of the expression construct. $P p u$-SL2 is expressed under the control of the C. elegans U2.3 snRNA promoter, and $s l s-1$ terminator in strains PE535/536. (B) Detection of trans-splicing of $P p u$-SL2 RNA. Total RNA prepared from strains PE535/536 was reverse transcribed. cDNA synthesis was tested by amplification of act-1; trans-splicing of Ppu-SL2 to Y67H2A.5 transcripts was detected using a gene-specific primer in combination with the Ppu-SL2 primer. In a control reaction $(-\mathrm{RT})$, the reverse transcriptase was omitted from the cDNA synthesis reaction. PCR reactions were analyzed by agarose gel electrophoresis (shown in inversed colors), and PCR products were analyzed by sequencing. The $5^{\prime}$ end of the sequence including the Ppu-SL2 primer sequence is shown. Critical residues that allow the distinction between transsplicing to Ppu-SL2 and to SL1 or SL2 are indicated with arrows, and the Y67H2A.5 transcript region is boxed black, with the beginning of the open reading frame in italics. The SL2 splice acceptor site is indicated $(\Delta)$. (C) Trans-splicing of Ppu-SL2 to tct-1, rla-1, and rps-3 transcripts in PE536. Trans-splicing to SL1 or SL2 or Ppu-SL2 RNAs was detected using gene-specific primers in combination with SL1, SL2, or Ppu-SL2 primers. Control PCR amplifications were performed with mock cDNA preparations performed without reverse transcriptase $(-\mathrm{RT})$. Amplification products were identified by DNA sequencing. $M$ indicates marker lane, in base pairs (bp). The acceptor sites for SL2 and Ppu-SL2 are indicated in the genomic tct-1 and rla-1 sequences $(\boldsymbol{\Lambda})$.

one derived from Ppu-SL2 we were able to amplify a product that when sequenced was shown to correspond to the addition of $P p u$-SL2 to the previously determined SL acceptor site of Y67H2A.5 (Fig. 2B). As critical Ppu-SL2specific residues are not part of the primer, the sequence analysis reliably identifies the participation of Ppu-SL2 in trans-splicing. Thus, $P p u$-SL2 was able to participate in SL 
trans-splicing in C. elegans and compete with endogenous C. elegans SL2 RNAs.

To further investigate the specificity of Ppu-SL2 for SL acceptor sites, we applied the same assay to a set of genes known to show a very high degree of specificity for the addition of either SL1 or SL2 SLs at their $5^{\prime}$ ends. The tct-1 and rla-1 genes show close to $100 \%$ specificity for transsplicing to C. elegans SL2, whereas the rps-3 gene shows a similar degree of specificity for SL1 (T Blumenthal, pers. comm.). We were able to detect PCR products corresponding to the addition of $P p u$-SL2 to both tct-1 and rla-1, but not to $r p s-3$, indicating that $P p u$-SL2 was specifically added only to the $5^{\prime}$ ends of SL2 trans-spliced transcripts (Fig. 2C). We also tested the addition of Ppu-SL2 to a pair of genes that form the upstream and downstream genes of an operon: $k u p-1$ and $p k c-1$, respectively (Spieth et al. 1993). Like most upstream genes, $k u p-1$ is predominantly transspliced to SL1, and we were unable to detect splicing of Ppu-SL2 to kup-1 (Fig. 3). Trans-splicing of pkc-1 occurs at two alternative acceptor sites, one of which splices mainly to SL2, whereas the other occurs largely via SL1. Consistent with our previous results, we only found addition of $P p u$ SL2 to the SL2 acceptor site of $p k c-1$ (Fig. 3).

Taken together, our results demonstrate that Ppu-SL2 RNA is able to participate in SL trans-splicing reactions in C. elegans with a specificity that indicates it interacts with the SL2 splicing machinery of C. elegans. Thus, it seems likely that the sequence similarity between $P p u$-SLs and the C. elegans SL2s is reflective of functional conservation.

\section{DISCUSSION}

The identification of SL trans-splicing in another member of a basal nematode clade reinforces the hypothesis that this process was present in the last common ancestor of all extant nematodes, and is consistent with the fact that it has been identified in all members of the phylum that have been characterized in molecular detail.

Our previous study showed that SL RNAs in T. spiralis exhibited a high degree of molecular heterogeneity and did not share strong sequence similarity to previously identified SL RNAs from the Rhabditida clade (Pettitt et al. 2008). Somewhat surprisingly, given that it is located in the same basal clade as T. spiralis (Fig. 1A), the SL RNAs that we have identified from $P$. punctatus do not closely resemble any of those from $T$. spiralis. Rather they show clear similarity to the SL2 family derived from the much more distantly related Rhabditina nematodes. Given the biased nature of the strategies that we employed to identify SLs following the identification of $P p u$-SL1, it is very likely that we have only sampled a subset of the complement of SL RNA genes in this nematode. It is possible that the

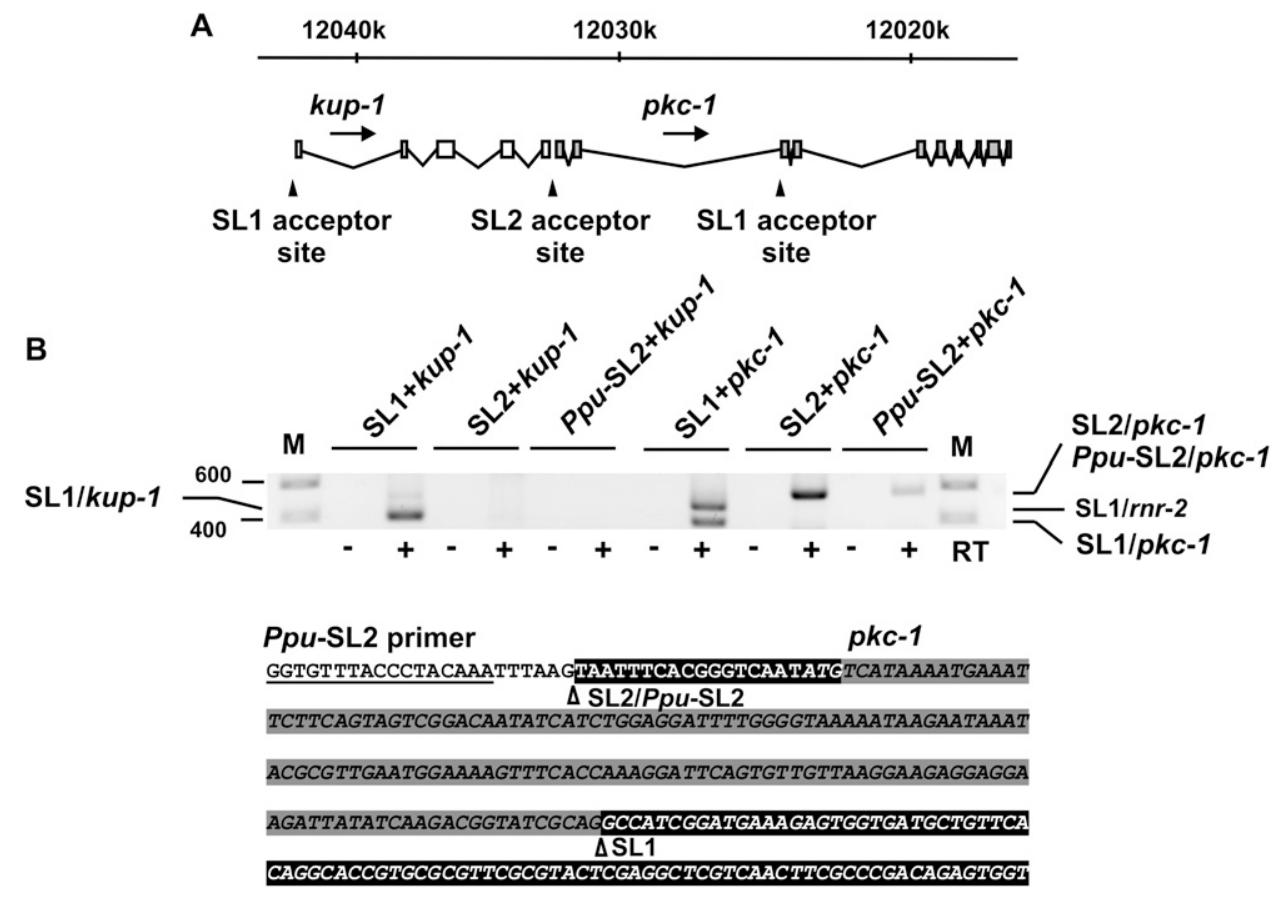

FIGURE 3. $P p u$-SL2 is specifically trans-spliced to the SL2 acceptor site of $p k c-1$. (A) Schematic showing the first $(k u p-1)$ and second ( $p k c-1)$ gene in operon CEOP5312 with SL1 and SL2 splice acceptor sites. Note that the SL2 acceptor site and the two first exons of pkc-1 are not currently annotated in WormBase. (B) Trans-splicing of Ppu-SL2, SL1, and SL2 RNAs to kup-1 and pkc-1 in PE536 was detected using the combination of gene-specific primers with SL1, SL2, or Ppu-SL2 primers indicated. Control PCR amplifications were performed with mock cDNA preparations performed without reverse transcriptase $(-\mathrm{RT})$. Amplification products were identified by DNA sequencing. The SL1 and SL2 acceptor sites $(\Delta)$ are indicated in the sequence of the $P p u$-SL2/pkc-1 fragment shown. The first two exons are boxed in gray; the following exons, in black. The beginning of the open reading frame is italicized. $\mathrm{M}$ indicates marker lane, in base pairs (bp). 
P. punctatus SL RNA gene family shows much greater diversity than that represented by the genes we report here. However, we have carried out exhaustive searches of the $T$. spiralis draft genome and failed to identify any SL RNA genes that resemble those from $P$. punctatus. Thus it seems likely that there are differences in the complements of SL RNAs between these two nematodes, underlining the importance of investigating this process in multiple representatives of a given taxon.

The identification of $P$. punctatus SL RNAs further emphasizes the diversity of SL RNAs in T. spiralis. Similarly diverse SL RNAs have recently been found in other groups of animals (Marletaz et al. 2008; Derelle et al. 2010; Douris et al. 2010), and it seems that the high degree of sequence conservation found between the majority of known nematode SLs is atypical. Recent work has shown that there are sequence constraints in the majority of nematode SLs associated with efficient translation of tri-methyl guanosine-capped RNAs generated by SL trans-splicing (Wallace et al. 2010). While the $P$. punctatus SLs conform to these sequence constraints, this is not the case for most $T$. spiralis SLs, reflecting their greater molecular heterogeneity. Those T. spiralis SLs that do conform also stimulate translation in vitro, although with lower efficiency than C. elegans SL1 (Wallace et al. 2010). The functional significance of these observations is unclear. However, since the SL RNA complements from many nonnematodes are characterized by high sequence diversity, a consensus picture is emerging whereby the acquisition of SL RNA genes (via mutation of snRNA genes) is followed by gene duplication and rapid sequence evolution. If this is how SL trans-splicing has arisen, the situation in T. spiralis would appear to represent the preservation of the ancestral nematode SL RNA state. It will thus be important to obtain a broader sampling of $P$. punctatus SL RNAs to determine whether this nematode has more diverse SL RNAs of the kind found in T. spiralis.

The assignment of the P. punctatus SL RNAs to the SL2 family was initially based upon sequence similarity; however, the outcome of expressing one of the P. punctatus SL RNAs in C. elegans provides further support for this classification. The fact that $P p u$-SL2 could be detected at the $5^{\prime}$ ends of $C$. elegans mRNAs showed that the RNA could be recognized by the $C$. elegans trans-splicing machinery. More importantly, this SL RNA was detected only at the $5^{\prime}$ ends of mRNAs derived from downstream genes in operons, indicating that it was specifically recognized by the SL2 splicing machinery, as predicted on the basis of sequence similarity.

We were unable to detect SL trans-splicing of Ppu-SL3.1 in similar assays in C. elegans and were also unable to detect the expression of this RNA in C. elegans, presumably because it was unstable. This indicates that there are functional differences between the P. punctatus SL RNAs. It is noteworthy that Ppu-SL3.1 does not contain elements in the third stem-loop essential for SL2 trans-splicing in C. elegans (Evans and Blumenthal 2000) and so may not be capable of forming a ribonucleoprotein complex important for stability in C. elegans. This sequence is also lacking in most $T$. spiralis SL RNAs (it is only present in $t s l r-2$, and closely related sequences are found in tslr-6, -11, and -12) (Pettitt et al. 2008), but this is not indicative of significant similarity between Ppu-SL3 RNAs and T. spiralis SL RNAs. Since $P$ pu-SL3.1 can be detected at the $5^{\prime}$ ends of $P$. punctatus mRNAs, it is clearly able to form a functional ribonucleopotein complex in that nematode.

The fact that at least one of the Ppu-SL RNAs can participate in SL2 trans-splicing in C. elegans suggests the possibility that these SL RNAs might also be involved in SL trans-splicing onto mRNAs derived from downstream genes in operons in $P$. punctatus. Operons have not been identified in any nematode that lies outside the Rhabditida group, although this may be a reflection of the relative paucity of molecular information available. The identification of SL2-like SL RNAs in P. punctatus offers a potential experimental route to identify and characterize putative operons in this phylogenetically important nematode.

Previous surveys of SL trans-splicing have indicated that the SL2 family is confined to the Rhabditina clade (Guiliano and Blaxter 2006), where it is associated with the SL transsplicing of mRNAs derived from downstream genes in operons. In nematodes that lie outside the Rhabditina, although operons exist, the mRNAs derived from downstream genes are trans-spliced to SL1 and SL1-like SLs, and SL2 RNAs appear to be absent from several species for which draft genome sequences are available (Guiliano and Blaxter 2006). This led to the hypothesis that SL2 RNAs are an innovation specific to the Rhabditina. Our identification of a group of SL2-like SL RNAs in P. punctatus throws into question the hypothesis that the SL2 family is a relatively recent evolutionary acquisition. Based on our current understanding of the phylogenetic position of $P$. punctatus and Rhabditina nematodes, their last common ancestor was the founder of the nematode phylum. Thus the presence of SL2-like molecules in these distantly related nematodes opens up the possibility for a more ancient origin for this family. If this is the case, then there must have been multiple independent losses of SL2-like molecules to explain the current distribution of SL2 family members. Recent surveys of EST data from the nonrhabditine Aphelenchus avenae have identified SL2-like SLs, providing further evidence that this family is more broadly distributed than previously determined (Reardon et al. 2010).

An alternative explanation for the presence of the SL2like SL RNAs in P. punctatus is that they were acquired via horizontal transmission from the rhabditine nematodes that likely constitute their prey in the wild. This would also explain their absence from T. spiralis. However, we are not aware of any examples of horizontal gene transfer between nematodes, but transfer of genes for plant cell wall degrading enzymes between bacteria and plant parasitic 
nematodes is now accepted (Bird et al. 2009). It is also possible that the similarities between the P. punctatus SL2like and C. elegans SL2s are due to independent, convergent events, although the constraints that might lead to the acquisition of similar sequences are unknown. Ultimately, however, it will be necessary to obtain a more comprehensive picture of the SL RNA complement in P. punctatus, and in other dorylaimid nematodes, in order to distinguish between these possibilities and to obtain a more complete picture of the evolution of nematode SL trans-splicing.

\section{MATERIALS AND METHODS}

\section{Nematode culture}

Rhabditis dolichura were grown and maintained on NGM agar seeded with a lawn of the Escherichia coli strain OP 50 and incubated at $20^{\circ} \mathrm{C}$. P. punctatus was a gift from Gaetan Borgonie (Ghent University). P. punctatus was grown on soil agar prepared as described by Small and Evans (1981), supplemented with nystatin $(62.5 \mathrm{KU} / \mathrm{mL})$ and cholesterol $(5 \mathrm{mg} / \mathrm{mL})$. One day prior to the addition of $P$. punctatus, the prey nematodes, $R$. dolichura, were added to the soil agar plates. Incubation was at $20^{\circ} \mathrm{C}$. Cultures were incubated for about 4-6 wk, and the adult $P$. punctatus were harvested by washing plates with M9 medium.

\section{RNA isolation}

Isolation of total RNA was carried out using Tri Reagent (Sigma), following a protocol described elsewhere (Keall et al. 2007).

\section{Cloning of cDNAs and determination of cDNA ends}

cDNA synthesis from C. elegans total RNA was carried out using either random hexamers or an oligo-dT primer and M-MLV reverse transcriptase. Cloning of $P$. punctatus cDNAs and determination of the $5^{\prime}$ and $3^{\prime}$ cDNA ends were done using total RNA and the GeneRacer kit (Invitrogen). The gene-specific primers used are given in Supplemental Table 2. cDNAs were amplified by PCR and cloned into pGEM T-Easy (Promega) or pCR4-TOPO (Invitrogen) plasmid vectors, and the resulting DNA inserts sequenced by the University of Dundee Sequencing Service. $P$. punctatus sequence data linked to this study have been deposited in GenBank with accession nos. GU936298-GU936311.

\section{SL RNA 3' end mapping.}

The $P$. punctatus SL RNA 3' ends were determined essentially as described previously (Pettitt et al. 2008).

\section{Amplification of $P$. punctatus genomic DNA.}

Normally, 1-10 animals were picked from soil agar plates and transferred into lysis buffer (20 mM Tris [pH 7.5], 50 mM EDTA, $200 \mathrm{mM} \mathrm{NaCl}, 0.5 \%$ SDS$)$. Proteinase $\mathrm{K}(10 \mathrm{mg} / \mathrm{mL})$ was then added to a concentration of $100 \mu \mathrm{g} / \mathrm{mL}$, and the mixtures were incubated for $1 \mathrm{~h}$ at $60^{\circ} \mathrm{C}$, followed by $15 \mathrm{~min}$ at $95^{\circ} \mathrm{C}$. PCR was carried out with the primers given in Supplemental Table 2 using the Expand Long Template PCR Kit (Roche). For DNA sequencing, fragments were excised from agarose gels, isolated using the
QIAquick gel extraction kit (Qiagen), and cloned into pGEM-T easy (Promega) or pCR4-TOPO (Invitrogen).

\section{Expression of a $P$. punctatus SL RNA in $C$. elegans}

The C. elegans U2-3 promoter was amplified from wild-type genomic DNA using the primers KF99 and KF101 (all primers are listed in Supplemental Table 2). PpuSL2_U and PpuSL2_D are partially overlapping and encode the $P p u$-SL2 RNA sequence. CeSL13U_U and CeSL13_U are also partially overlapping and encode the $3^{\prime}$ untranslated region (3'UTR) of the C. elegans SL1 gene. The construct composed of the C. elegans U2-3 promoter, $P p u$-SL2 RNA, and the 3'UTR of the C. elegans SL1 gene was created in a single PCR reaction utilizing the oligonucleotides KF99 and CeSL13U-pr. The resulting product was cloned into pGEM-T easily and sequenced to verify that it contained no mutations. The plasmid was co-injected with pTG96 to generate transgenic $C$. elegans strains using standard procedures (Mello and Fire 1995). Multiple lines were obtained, and two (PE535 and PE536) were chosen for RT-PCR analysis. After RNA preparation and reverse transcription, trans-splicing was analyzed by PCR using combinations of SL-specific and gene-specific primers (Supplemental Table 2). PCR reactions were analyzed by agarose gel electrophoresis and the results were visualized by staining with ethidium bromide. The grayscale pictures in Figures 2 and 3 are inverted for increased clarity.

\section{SUPPLEMENTAL MATERIAL}

Supplemental material can be found at http://www.rnajournal.org.

\section{ACKNOWLEDGMENT}

We thank Gaetan Borgonie for providing us with P. punctatus, Fabien Javerliat for identifying the first $P$. punctatus cDNA, and Tom Blumenthal for personal communications and his interest in this project. A.K. was supported by the EU Erasmus exchange program. This work was supported in part by a Wellcome Trust project grant (WT076220).

\section{NOTE ADDED IN PROOF}

We have identified additional upstream sequences of the Ppu-vha-1 gene including a typical trans-splice acceptor site at the correct location (GenBank entry GU936299) (see Supplemental Table 3).

Received March 9, 2010; accepted May 14, 2010.

\section{REFERENCES}

Bird DM, Williamson VM, Abad P, McCarter J, Danchin EGJ, Castagnone-Sereno P, Opperman CH. 2009. The genomes of root-knot nematodes. Annu Rev Phytopathol 47: 333-351.

Blumenthal T. 2005. Trans-splicing and operons. In Wormbook (ed. The C.elegans Research Community). WormBook. doi: 10.1895/ wormbook.1.5.1. http://www.wormbook.org.

Blumenthal T, Evans D, Link CD, Guffanti A, Lawson D, ThierryMieg J, Thierry-Mieg D, Chiu WL, Duke K, Kiraly M, et al. 2002. A global analysis of Caenorhabditis elegans operons. Nature 417: 851-854. 
Bürglin T. 2008. Evolution of hedgehog and hedgehog-related genes, their origin from Hog proteins in ancestral eukaryotes and discovery of a novel Hint motif. BMC Genomics 9: 127.

Davis RE. 1997. Surprising diversity and distribution of spliced leader RNAs in flatworms. Mol Biochem Parasitol 87: 29-48.

Davis RE, Hodgson S. 1997. Gene linkage and steady state RNAs suggest trans-splicing may be associated with a polycistronic transcript in Schistosoma mansoni. Mol Biochem Parasitol 89: 25-39.

Derelle R, Momose T, Manuel M, Da Silva C, Wincker P, Houliston E. 2010. Convergent origins and rapid evolution of spliced leader trans-splicing in Metazoa: Insights from the Ctenophora and Hydrozoa. RNA 16: 696-707.

Douris V, Telford MJ, Averof M. 2010. Evidence for multiple independent origins of trans-splicing in Metazoa. Mol Biol Evol 27: 684-693.

Evans D, Blumenthal T. 2000. Trans splicing of polycistronic Caenorhabditis elegans Pre-mRNAs: Analysis of the SL2 RNA. Mol Cell Biol 20: 6659-6667.

Evans D, Zorio D, MacMorris M, Winter CE, Lea K, Blumenthal T. 1997. Operons and SL2 trans-splicing exist in nematodes outside the genus Caenorhabditis. Proc Natl Acad Sci 94: 9751-9756.

Ferguson KC, Rothman JH. 1999. Alterations in the conserved SL1 trans-spliced leader of Caenorhabditis elegans demonstrate flexibility in length and sequence requirements in vivo. Mol Cell Biol 19: $1892-1900$.

Guiliano DB, Blaxter ML. 2006. Operon conservation and the evolution of trans-splicing in the Phylum Nematoda. PLoS Genet 2: e198. doi: 10.1371/journal.pgen.0020198.

Hastings KEM. 2005. SL trans-splicing: Easy come or easy go? Trends Genet 21: 240-247.

Heger P, Marin B, Schierenberg E. 2009. Loss of the insulator protein CTCF during nematode evolution. BMC Mol Biol 10: 84 .

Holterman $M$, van der Wurff $A$, van den Elsen S, van Megen $H$, Bongers T, Holovachov O, Bakker J, Helder J. 2006. Phylum-wide analysis of SSU rDNA reveals deep phylogenetic relationships among nematodes and accelerated evolution toward crown clades. Mol Biol Evol 23: 1792-1800.

Keall R, Whitelaw S, Pettitt J, Müller B. 2007. Histone gene expression and histone mRNA $3^{\prime}$ end structure in Caenorhabditis elegans. BMC Mol Biol 8: 51.

Lall S, Friedman CC, Jankowska-Anyszka M, Stepinski J, Darzynkiewicz E, Davis RE. 2004. Contribution of trans-splicing, 5' -leader length, cap-poly(A) synergism, and initiation factors to nematode translation in an Ascaris suum embryo cell-free system. J Biol Chem 279: 45573-45585.

Lavrov DV, Brown WM. 2001. Trichinella spiralis mtDNA: A nematode mitochondrial genome that encodes a putative ATP8 and normally structured tRNAS and has a gene arrangement relatable to those of coelomate metazoans. Genetics 157: 621-637.

Marletaz F, Gilles A, Caubit X, Perez Y, Dossat C, Samain S, Gyapay G, Wincker P, Le PY. 2008. Chaetognath transcriptome reveals ancestral and unique features among bilaterians. Genome Biol 9: R94.

Matsumoto J, Dewar K, Wasserscheid J, Macmil SL, Wiley GB, Roe BA, Zeller RW, Satou Y, and Hastings KEM. 2010. Highthroughput sequence analysis of Ciona intestinalis SL trans-spliced mRNAs: Alternative expression modes and gene function correlates. Genome Research 20: 636-645.
Mattaj IW. 1988. U snRNP assembly and transport. In Structure and function of major and minor small nuclear ribonucleoprotein particles (ed. ML Birnstiel), pp. 100-114. Springer, New York.

Meldal BHM, Debenham NJ, De Ley P, De Ley IT, Vanfleteren JR, Vierstraete AR, Bert W, Borgonie G, Moens T, Tyler PA, et al. 2007. An improved molecular phylogeny of the Nematoda with special emphasis on marine taxa. Mol Phylogenet Evol 42: 622-636.

Mello C, Fire A. 1995. DNA transformation. In Caenorhabditis elegans: Modern biological analysis of an organism (ed. HF Epstein and DC Shakes), pp. 451-482. Academic, San Diego, CA.

Pettitt J, Müller B, Stansfield I, Connolly B. 2008. Spliced leader transsplicing in the nematode Trichinella spiralis uses highly polymorphic, noncanonical spliced leaders. RNA 14: 760-770.

Pouchkina-Stantcheva NN, Tunnacliffe A. 2005. Spliced leader RNAmediated trans-splicing in Phylum Rotifera. Mol Biol Evol 22: 1482-1489.

Reardon W, Chakrabortee S, Campos Pereira T, Tyson T, Banton M, Dolan K, Culleton B, Wise M, Burnell A, Tunnacliffe A. 2010. Expression profiling and cross-species RNA interference (RNAi) of desiccation-induced transcripts in the anhydrobiotic nematode Aphelenchus avenae. BMC Mol Biol 11: 6.

Satou Y, Hamaguchi M, Takeuchi K, Hastings KEM, Satoh N. 2006. Genomic overview of mRNA 5'-leader trans-splicing in the ascidian Ciona intestinalis. Nucleic Acids Res 34: 3378-3388.

Schulze J, Schierenberg E. 2009. Embryogenesis of Romanomermis culicivorax: An alternative way to construct a nematode. Dev Biol 334: $10-21$.

Small RH, Evans AAF. 1981. Experiments on population growth of the predatory nematode Prionchulus punctatus in laboratory culture with observations on life history. Rev Nematol 4: 261-270.

Spieth J, Brooke G, Kuersten S, Lea K, Blumenthal T. 1993. Operons in C. elegans: Polycistronic mRNA precursors are processed by transsplicing of SL2 to downstream coding regions. Cell 73: 521-532.

Stover NA, Steele RE. 2001. Trans-spliced leader addition to mRNAs in a cnidarian. Proc Natl Acad Sci 98: 5693-5698.

Sutton RE, Boothroyd JC. 1986. Evidence for trans splicing in trypanosomes. Cell 47: 527-535.

Tessier LH, Keller M, Chan RL, Fournier R, Weil JH, Imbault P. 1991. Short leader sequences may be transferred from small RNAs to pre-mature mRNAs by trans-splicing in Euglena. EMBO J 10: $2621-2625$

Wallace A, Filbin ME, Veo B, McFarland C, Stepinski J, JankowskaAnyszka M, Darzynkiewicz E, Davis RE. 2010. The nematode eIF4E/G complex works with a trans-spliced leader stem-loop to enable efficient translation of trimethylguanosine-capped RNAs. Mol Cell Biol 30: 1958-1970.

Zayas RM, Bold TD, Newmark PA. 2005. Spliced-leader trans-splicing in freshwater planarians. Mol Biol Evol 22: 2048-2054.

Zeiner GM, Sturm NR, Campbell DA. 2003. The Leishmania tarentolae spliced leader contains determinants for association with polysomes. J Biol Chem 278: 38269-38275.

Zhang H, Lin S. 2009. Retrieval of missing spliced leader in dinoflagellates. PLOS ONE 4: e4129. doi: 10.1371/journal.pone.0004129.

Zhang H, Hou Y, Miranda L, Campbell DA, Sturm NR, Gaasterland T, Lin S. 2007. Spliced leader RNA trans-splicing in dinoflagellates. Proc Natl Acad Sci 104: 4618-4623.

Zuker M. 2003. Mfold web server for nucleic acid folding and hybridization prediction. Nucleic Acids Res 31: 3406-3415. 

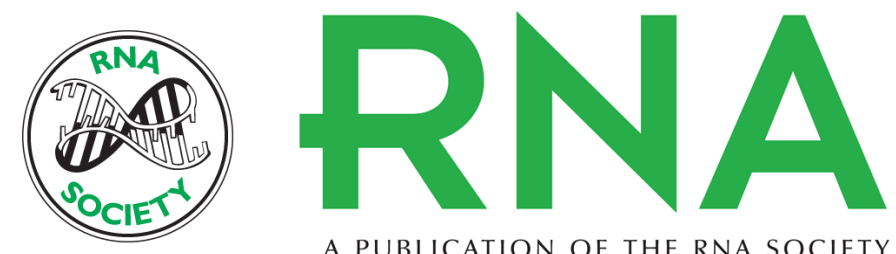

A PUBLICATION OF THE RNA SOCIETY

\section{SL2-like spliced leader RNAs in the basal nematode Prionchulus punctatus : New insight into the evolution of nematode SL2 RNAs}

Neale Harrison, Andreas Kalbfleisch, Bernadette Connolly, et al.

RNA 2010 16: 1500-1507 originally published online June 21, 2010

Access the most recent version at doi:10.1261/rna.2155010

\section{Supplemental http://rnajournal.cshlp.org/content/suppl/2010/06/08/rna.2155010.DC1 \\ Material}

References This article cites 36 articles, 11 of which can be accessed free at: http://rnajournal.cshlp.org/content/16/8/1500.full.html\#ref-list-1

\section{License}

Email Alerting Receive free email alerts when new articles cite this article - sign up in the box at the Service top right corner of the article or click here.

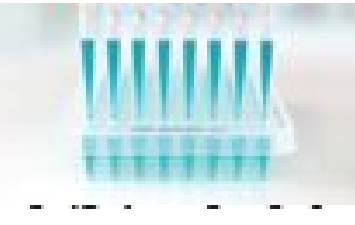

Providing Precise Solutions for your research.

To subscribe to $R N A$ go to:

http://rnajournal.cshlp.org/subscriptions 